\title{
Relativistic mean-field description of the dynamics of giant resonances
}

\author{
D. Vretenar ${ }^{a}$, P. Ring ${ }^{b}$, G.A. Lalazissis ${ }^{b}$, and N.Paar ${ }^{a}$ \\ a Physics Department, Faculty of Science, \\ University of Zagreb, Croatia \\ b Physik-Department der Technischen Universität München, \\ D-85748 Garching, Germany
}

September 28, 2018

\begin{abstract}
The relativistic mean-field theory provides a framework in which the nuclear many-body problem is described as a self-consistent system of nucleons and mesons. In the mean-field approximation, the self-consistent time evolution of the nuclear system describes the dynamics of collective motion: nuclear compressibility from monopole resonances, regular and chaotic dynamics of isoscalar and isovector collective vibrations.
\end{abstract}

\section{The relativistic mean field model}

Relativistic mean-field (RMF) models have been successfully applied in calculations of nuclear matter and properties of finite nuclei throughout the periodic table. In the self-consistent mean-field approximation, detailed calculations have been performed for a variety of nuclear structure phenomena [1]]. 
In this work we present applications of RMF to the dynamics of collective vibrations in spherical nuclei. In relativistic quantum hadrodynamics [2], the nucleus is described as a system of Dirac nucleons which interact through the exchange of virtual mesons and photons. The Lagrangian density of the model is

$$
\begin{aligned}
\mathcal{L}= & \bar{\psi}(i \gamma \cdot \partial-m) \psi+\frac{1}{2}(\partial \sigma)^{2}-U(\sigma) \\
& -\frac{1}{4} \Omega_{\mu \nu} \Omega^{\mu \nu}+\frac{1}{2} m_{\omega}^{2} \omega^{2}-\frac{1}{4} \overrightarrow{\mathrm{R}}_{\mu \nu} \overrightarrow{\mathrm{R}}^{\mu \nu}+\frac{1}{2} m_{\rho}^{2} \vec{\rho}^{2}-\frac{1}{4} \mathrm{~F}_{\mu \nu} \mathrm{F}^{\mu \nu} \\
& -g_{\sigma} \bar{\psi} \sigma \psi-g_{\omega} \bar{\psi} \gamma \cdot \omega \psi-g_{\rho} \bar{\psi} \gamma \cdot \vec{\rho} \vec{\tau} \psi-e \bar{\psi} \gamma \cdot A \frac{\left(1-\tau_{3}\right)}{2} \psi .
\end{aligned}
$$

The Dirac spinor $\psi$ denotes the nucleon with mass $m . m_{\sigma}, m_{\omega}$, and $m_{\rho}$ are the masses of the $\sigma$-meson, the $\omega$-meson, and the $\rho$-meson, and $g_{\sigma}, g_{\omega}$, and $g_{\rho}$ are the corresponding coupling constants for the mesons to the nucleon. $U(\sigma)$ denotes the nonlinear $\sigma$ self-interaction, and $\Omega^{\mu \nu}, \vec{R}^{\mu \nu}$, and $F^{\mu \nu}$ are field tensors [田, 2]. The coupled equations of motion are derived from the Lagrangian density (迎). The Dirac equation for the nucleons:

$$
\begin{aligned}
i \partial_{t} \psi_{i}= & {\left[\boldsymbol{\alpha}\left(-i \boldsymbol{\nabla}-g_{\omega} \boldsymbol{\omega}-g_{\rho} \vec{\tau} \overrightarrow{\boldsymbol{\rho}}-e \frac{\left(1-\tau_{3}\right)}{2} \boldsymbol{A}\right)+\beta\left(m+g_{\sigma} \sigma\right)\right.} \\
& \left.+g_{\omega} \omega_{0}+g_{\rho} \vec{\tau} \vec{\rho}_{0}+e \frac{\left(1-\tau_{3}\right)}{2} A_{0}\right] \psi_{i}
\end{aligned}
$$

and the Klein-Gordon equations for the mesons:

$$
\begin{aligned}
\left(\partial_{t}^{2}-\Delta+m_{\sigma}^{2}\right) \sigma & =-g_{\sigma} \rho_{s}-g_{2} \sigma^{2}-g_{3} \sigma^{3} \\
\left(\partial_{t}^{2}-\Delta+m_{\omega}^{2}\right) \omega_{\mu} & =g_{\omega} j_{\mu} \\
\left(\partial_{t}^{2}-\Delta+m_{\rho}^{2}\right) \vec{\rho}_{\mu} & =g_{\rho} \vec{j}_{\mu} \\
\left(\partial_{t}^{2}-\Delta\right) A_{\mu} & =e j_{\mu}^{\mathrm{em}} .
\end{aligned}
$$

In the relativistic mean-field approximation, the nucleons described by singleparticle spinors $\psi_{i}$ are assumed to form the A-particle Slater determinant $|\Phi\rangle$, and to move independently in the classical meson fields. The sources of the fields, i.e. densities and currents, are calculated in the no-sea approximation [3]: the scalar density: $\rho_{\mathrm{s}}=\sum_{i=1}^{A} \bar{\psi}_{i} \psi_{i}$, the isoscalar baryon current: 
$j^{\mu}=\sum_{i=1}^{A} \bar{\psi}_{i} \gamma^{\mu} \psi_{i}$, the isovector baryon current: $\vec{j}^{\mu}=\sum_{i=1}^{A} \bar{\psi}_{i} \gamma^{\mu} \vec{\tau} \psi_{i}$, the electromagnetic current for the photon-field: $j_{\mathrm{em}}^{\mu}=\sum_{i=1}^{A} \bar{\psi}_{i} \gamma^{\mu} \frac{1-\tau_{3}}{2} \psi_{i}$. The summation is over all occupied states in the Slater determinant $|\Phi\rangle$. Negative-energy states do not contribute to the densities in the no-sea approximation of the stationary solutions. It is assumed that nucleon singleparticle states do not mix isospin.

The ground state of a nucleus is described by the stationary self-consistent solution of the coupled system of equations (2)-(6), for a given number of nucleons and a set of coupling constants and masses. The solution for the ground state specifies part of the initial conditions for the time-dependent problem. The dynamics of nuclear collective motion is analyzed in the framework of time-dependent relativistic mean-field model, which represents a relativistic generalization of the time-dependent Hartree-Fock approach. For a given set of initial conditions, i.e. initial values for the densities and currents, nuclear dynamics is described by the simultaneous evolution of $A$ single- particle Dirac spinors in the time-dependent mean fields. The equations (2) -(6) are equivalent to the equation of motion for the one-body density operator $\hat{\rho}=\hat{\rho}(t)$

$$
i \hbar \frac{\partial}{\partial t} \hat{\rho}=\left[h_{D}, \hat{\rho}\right],
$$

with an initial condition for $\hat{\rho}$

$$
\hat{\rho}\left(t_{i n}\right)=\hat{\rho}_{i n} .
$$

$h_{D}$ is the single-nucleon Dirac hamiltonian defined in Eq. (2). Starting from the self-consistent solution that describes the ground-state of the nuclear system, initial conditions are defined to simulate excitations of giant resonances in experiments with electromagnetic or hadron probes. Frequencies of eigenmodes are determined from a Fourier analysis of dynamical quantities. In this microscopic model, self-consistent time-dependent mean-field calculations are performed for multipole excitations. An advantage of the timedependent approach is that no assumption about the nature of a particular mode of vibrations has to be made. Retardation effects for the meson fields are not included in the model, i.e. the time derivatives $\partial_{t}^{2}$ in the equations of motions for the meson fields are neglected. This is justified by the large masses in the meson propagators causing a short range of the corresponding meson exchange forces. Negative energy contributions are included implicitly 
in the time-dependent calculation, since the Dirac equation is solved at each step in time for a different basis set [3]. Negative energy components with respect to the original ground-state basis are taken into account automatically, even if at each time step the no-sea approximation is applied.

The description of nuclear dynamics as a time-dependent initial-value problem is intrinsically semi-classical, since there is no systematic procedure to derive the initial conditions that characterize the motion of a specific mode of the nuclear system. The theory can be quantized by the requirement that there exist time-periodic solutions of the equations of motion, which give integer multiples of Planck's constant for the classical action along one period [4. For giant resonances the time-dependence of collective dynamical quantities is actually not periodic, since generally giant resonances are not stationary states of the mean-field Hamiltonian. The coupling of the meanfield to the particle continuum allows for the decay of giant resonances by direct escape of particles. In the limit of small amplitude oscillations, however, the energy obtained from the frequency of the oscillation coincides with the excitation energy of the collective state. In Refs. [3, 4, 5] we have shown that the model reproduces experimental data on giant resonances in spherical nuclei.

\section{Monopole resonances and nuclear compress- ibility}

The study of isoscalar monopole resonances in nuclei provides an important source of information on the nuclear matter compression modulus $K_{\mathrm{nm}}$. This quantity is crucial in the description of properties of nuclei, supernovae explosions, neutron stars, and heavy-ion collisions. In principle the value of $K_{\mathrm{nm}}$ can be extracted from experimental energies of isoscalar monopole vibrations in nuclei (giant monopole resonances GMR). However, the complete experimental data set on isoscalar GMR does not limit the range of $K_{\mathrm{nm}}$ to better than $200-350 \mathrm{MeV}$. Microscopic calculations of GMR excitation energies might provide a more reliable approach to the determination of the nuclear matter compression modulus. Modern non-relativistic Hartree-Fock plus RPA calculations, using both Skyrme and Gogny effective interactions, indicate that the value of $K_{\mathrm{nm}}$ should be in the range $210-220 \mathrm{MeV}$. In rela- 
tivistic calculations on the other hand, both time-dependent and constrained RMF results indicate that empirical GMR energies are best reproduced by an effective force with $K_{\mathrm{nm}} \approx 250-270 \mathrm{MeV}$.

In Ref. [5] we have performed time-dependent and constrained RMF calculations for monopole giant resonances for a number of spherical closed shell nuclei, from ${ }^{16} \mathrm{O}$ to the heavy nucleus ${ }^{208} \mathrm{~Pb}$. For the effective Lagrangian we have used six parameter sets, which differ mostly by their prediction for $K_{\mathrm{nm}}$, but otherwise reproduce reasonably well experimental data on nuclear properties. The idea is to restrict the possible values of the nuclear matter compression modulus, on the basis of the excitation energies of giant monopole states calculated with different effective interactions. In addition to the four non-linear sets NL1, NL3, NL-SH and NL2, we have also included two older linear parametrizations, HS and L1. The sets NL1, NL-SH and NL2 have been extensively used in the description of properties of finite nuclei [1]. In order to bridge the gap between NL1 $\left(K_{\mathrm{nm}}=211.7 \mathrm{MeV}\right)$, and NL-SH $\left(K_{\mathrm{nm}}=355.0 \mathrm{MeV}\right)$, we have also included a new effective interaction NL3 [6] $\left(K_{\mathrm{nm}}=271.8 \mathrm{MeV}\right)$. This new parameter set provides an excellent description not only for the properties of stable nuclei, but also for those far from the valley of beta stability. From the energy spectra and transition densities calculated with these effective forces, it has been possible to study the connection between the incompressibility of nuclear matter and the breathing mode energy of spherical nuclei. For the isoscalar mode we have found an almost linear relation between the excitation energy of the monopole resonance and the nuclear matter compression modulus. For the determination of $K_{\mathrm{nm}}$ especially relevant are microscopic calculations of GMR excitation energies in heavy nuclei. The results of TD RMF calculations for ${ }^{208} \mathrm{~Pb}$ are displayed in Fig. 1: time-dependent monopole moments $\left\langle r^{2}(t)\right\rangle=\frac{1}{A}\left\langle\Phi(t)\left|r^{2}\right| \Phi(t)\right\rangle$ and the corresponding Fourier power spectra for the nonlinear effective interactions. As one would expect for a heavy nucleus, there is very little spectral fragmentation and a single mode dominates, at least for NL1 and NL3. The experimental excitation energy $13.7 \pm 0.3 \mathrm{MeV}$ is very close to the frequency of oscillations obtained with the NL3 parameter set: $14.1 \mathrm{MeV}$. The calculated excitation energy for the NL1 parameter set $\left(K_{\mathrm{nm}}=211.7 \mathrm{MeV}\right)$, is approximately $1 \mathrm{MeV}$ lower than the average experimental value. For the linear effective forces HS and L1 the oscillations are more anharmonic, and the monopole strength is located well above the experimental GMR energy. 
The effective interactions NL1 and NL3 seem to produce GMR excitation energies which are quite close to the experimental values. For these two parameter sets we have calculated the isoscalar giant monopole resonances in a number of closed-shell nuclei: ${ }^{40} \mathrm{Ca},{ }^{56} \mathrm{Ni},{ }^{100,114,132} \mathrm{Sn},{ }^{90,122} \mathrm{Zr},{ }^{146} \mathrm{Gd}$. The results are shown in Fig. 2. The energies of giant monopole states are determined from the Fourier spectra of the time-dependent monopole moments, and are displayed as function of the mass number. The NL1 excitation energies are systematically lower, but otherwise the two effective interactions produce very similar dependence on the mass number. The empirical curve $E_{x} \approx 80 A^{-1 / 3} \mathrm{MeV}$ is also included in the figure, and it follows very closely the excitation energies calculated with the NL3 parameter set. Similar results are obtained from constrained RMF calculations. Both methods indicate that, in the framework of relativistic mean field theory, the nuclear matter compression modulus $K_{\mathrm{nm}} \approx 250-270 \mathrm{MeV}$ is in reasonable agreement with the available data on spherical nuclei. This value is approximately $20 \%$ larger than the values deduced from recent non-relativistic density dependent Hartree-Fock calculations with Skyrme or Gogny forces. It should be also emphasized that the excitation energy of the isovector monopole resonance in ${ }^{208} \mathrm{~Pb}$, calculated with the NL3 effective force, is in excellent agreement with the experimental value $26 \pm 3 \mathrm{MeV}$.

\section{Nonlinear dynamics of giant resonances}

Atomic nuclei provide excellent examples of quantum systems in which the transition from regular to chaotic dynamics can be studied. Signatures of chaotic dynamics have been observed in correlations of nuclear level distributions, and in the microscopic and collective motion of the nuclear many-body system. Especially interesting in this respect are giant resonances: highly collective nuclear excitations whose properties, excitation energies and widths, nevertheless reflect the underlying microscopic dynamics. Theoretical studies have shown that regular collective modes coexists with chaotic single-nucleon motion: the adiabatic mean-field created by the nucleons averages out the random components of their motion. However, it has also been shown that important differences exists between the isoscalar and isovector collective modes.

In particular, we have studied isoscalar and isovector monopole oscilla- 
tions in spherical nuclei [7], but analogous considerations apply to higher multipolarities. In Fig. 3 results are shown of time-dependent relativistic meanfield calculations for isoscalar and isovector oscillations in ${ }^{208} \mathrm{~Pb}$. Calculations have been performed for the NL1 effective interaction. In the isoscalar case both proton and neutron densities are radially expanded, while for the isovector mode the proton density is initially compressed by the same amount. In Fig. 3a we plot the time series of the isoscalar monopole moment $\left\langle r^{2}(t)\right\rangle$, and in Fig. 3b the corresponding isovector moment $\left\langle r_{\mathrm{p}}^{2}(t)>-<r_{\mathrm{n}}^{2}(t)>\right.$ is displayed. The isoscalar mode displays regular undamped oscillations, while for the isovector mode strongly damped anharmonic oscillations are observed. On the right-hand panels we plot the corresponding Fourier power spectra. The Fourier spectrum of the isovector mode is strongly fragmented, but the main peaks are found in the energy region $25-30 \mathrm{MeV}$, in agreement with the experimental data. For the isoscalar mode, the time series of the monopole moment and the Fourier spectrum show that the oscillations of the collective coordinate are regular. On the other hand, the appearance of a broad spectrum of frequencies seems to indicate that the isovector oscillations are chaotic. For time-series that result from linear physical processes the Fourier analysis unfolds the characteristic frequencies which are invariants of the dynamics, i.e. they classify the dynamics. For nonlinear systems the corresponding analysis is somewhat more complicated.

We have performed the analysis of phase spaces reconstructed from timeseries of collective dynamical variables that characterize the isoscalar and isovector oscillations. For the reconstruction of the dynamics two principal quantities have to be determined: the time delay and the dimension of the phase space on which the attractor unfolds. The delays which define the time-lagged variables have been determined from the first minima of the average mutual information function: $27 \mathrm{fm} / \mathrm{c}$ for the isoscalar, and $13 \mathrm{fm} / \mathrm{c}$ for the isovector mode. For the embedding dimensions the method of false nearest neighbors has been used: $d_{E}=3$ (isoscalar mode), and $d_{E}=4$ (isovector mode). The reconstructed phase space can be represented by a recurrence plot. For the isoscalar mode the recurrence plot displays a pattern representative for regular oscillations, for the isovector mode it indicates non-stationarity [8]. If the dynamics of a system is deterministic, the ensemble of phase space trajectories converges towards an invariant subset of the phase space - the attractor. For chaotic dynamics the attractor has fractional dimension, whereas the dimension is integer for regular dynamics. The cor- 
relation dimension $D_{2}$ of the attractor can be numerically evaluated from the $\log$-log plot of the correlation integral $C_{2}(r)$ vs the distance $r$ in phase space, for a set of increasing dimensions of the phase space. As the embedding dimension increases, $D_{2}$ should saturate at a value equal to the attractor's correlation dimension (Fig. 4). For the isoscalar mode, for $d \geq 3$, the correlation dimension saturates at $D_{2}=2$; it does not saturate for the isovector mode, but slowly increases to some fractional value between 2 and 3 . The fractional dimension of the attractor would imply chaotic or stochastic dynamics.

The identification and quantification of the regular or chaotic dynamics can be also based on the evaluation of information-theoretic functionals. For the time-dependent one-body nucleon densities, we have calculated the von Neumann information entropy functionals. The Fourier analysis has shown that the entropy of the isoscalar mode contains the same information as the dynamical variable, but the structure is more complicated for the isovector mode. Giant resonances represent collective oscillations of densities with finite spatial extension, and therefore provide excellent physical examples for the analysis of systems that have spatial as well as temporal structure. For a nonlinear system in chaotic regime, the influence of spatial motion on temporal chaos cab be studied: what are the spatial correlations in a finite system that displays chaotic oscillations of a collective dynamical variable? These correlations have been described with a time-dependent conditional entropy defined from a two-body nucleon density, and the result is a high degree of two-body correlations for the isoscalar mode, and very little spatial correlation for the isovector oscillations of the nucleon density [8]. From the dynamical variables that characterize the proton and neutron distributions, we have calculated the average mutual information for the isoscalar and isovector modes (Fig. 5). This function quantifies the information that is contained in the dynamical variable of the neutron distribution, about the proton subsystem, and vice versa. The two curves that correspond to isoscalar and isovector oscillations, are plotted as functions of the size of the box in the linear embedding of the time-series. The average information that is contained in the collective dynamical variable of the proton density, about the neutron density, is more than a factor three larger for the isoscalar mode.

Time-series, Fourier spectra, phase-space plots, Poincaré sections, autocorrelation functions, and Lyapunov exponents [7] have also shown that the motion of the collective coordinate is regular for isoscalar oscillations, and 
that it becomes chaotic when initial conditions correspond to the isovector mode.

\section{References}

[1] P. Ring, Progr. Part. Nucl. Phys. 37 (1996) 193.

[2] B.D. Serot and J.D. Walecka, Int. J. Mod. Phys. E6 (1997) 515.

[3] D. Vretenar, H. Berghammer, and P. Ring, Phys. Lett. B319 (1993) 29; Nucl. Phys. A581 (1995) 679.

[4] P. Ring, D. Vretenar, and B. Podobnik, Nucl. Phys. A598 (1996) 107.

[5] D. Vretenar, G. A. Lalazissis, R. Behnsch, W. Pöschl and P. Ring, Nucl. Phys. A621 (1997) 853.

[6] G. A. Lalazissis, J. König and P. Ring, Phys. Rev. C 55 (1997) 540.

[7] D. Vretenar, P. Ring, G. A. Lalazissis and W. Pöschl, Phys. Rev. E 56 (1997) 6418.

[8] D. Vretenar, N. Paar, P. Ring, and G. A. Lalazissis submitted to Phys. Rev. E 


\section{Figure Captions}

Fig. 1 Time-dependent isoscalar monopole moments $<r^{2}>(t)$ and the corresponding Fourier power spectra for ${ }^{208} \mathrm{~Pb}$. The parameter sets are NL1, NL3, NL-SH and NL2.

Fig. 2 Excitation energies of isoscalar giant monopole resonances in spherical nuclei as function of the mass number. The effective interactions are: NL1 (squares) and NL3 (circles). The solid curve corresponds to the empirical relation $\approx 80 A^{-1 / 3} \mathrm{MeV}$.

Fig. 3 Time-dependent isoscalar and isovector monopole moments for ${ }^{208} \mathrm{~Pb}$.

Fig. 4 Correlation dimension $D_{2}$ as function of embedding dimension, for isoscalar and isovector oscillations.

Fig. 5 Mutual information between the time-dependent mean square radii of the proton and neutron density distributions. 


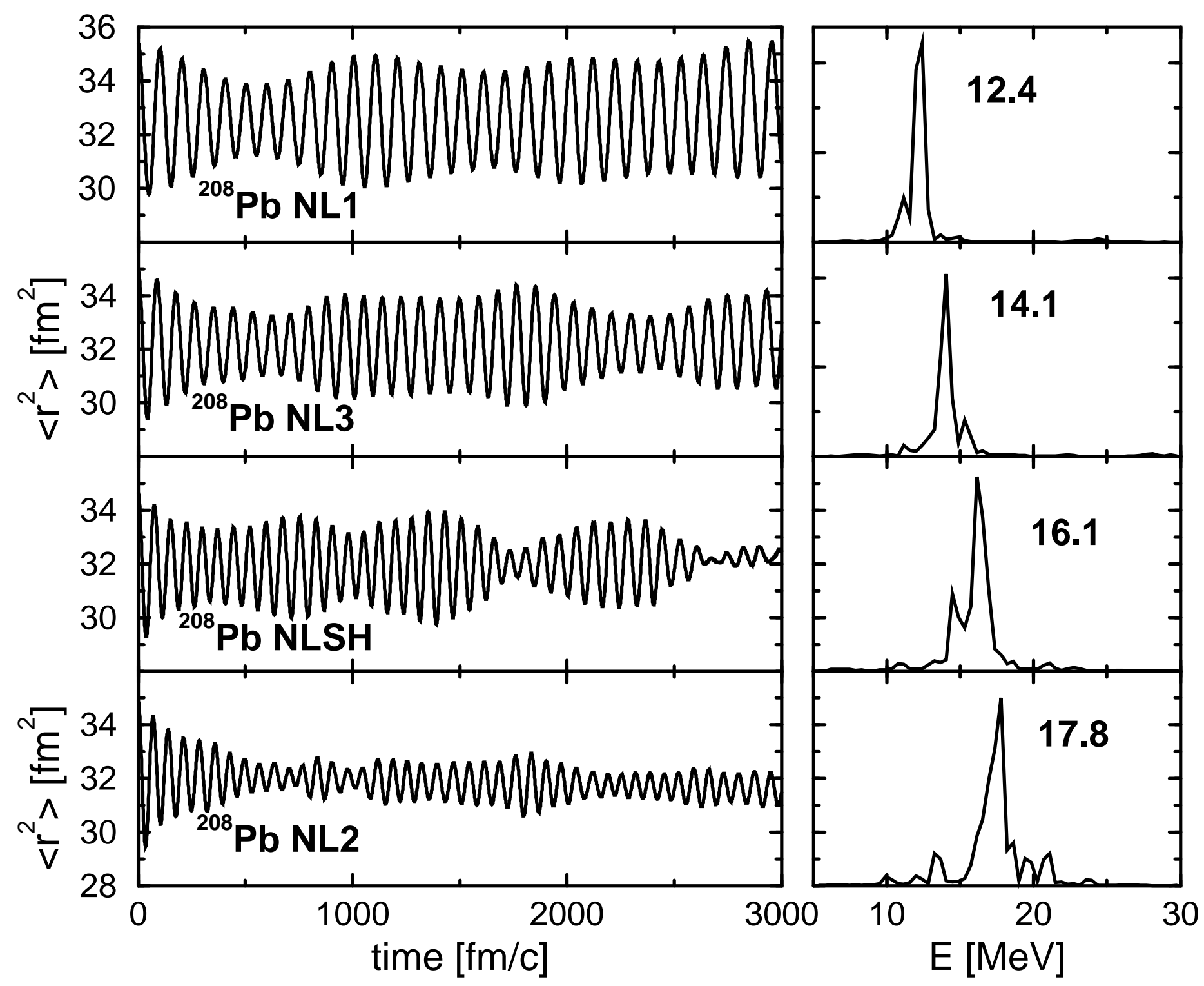




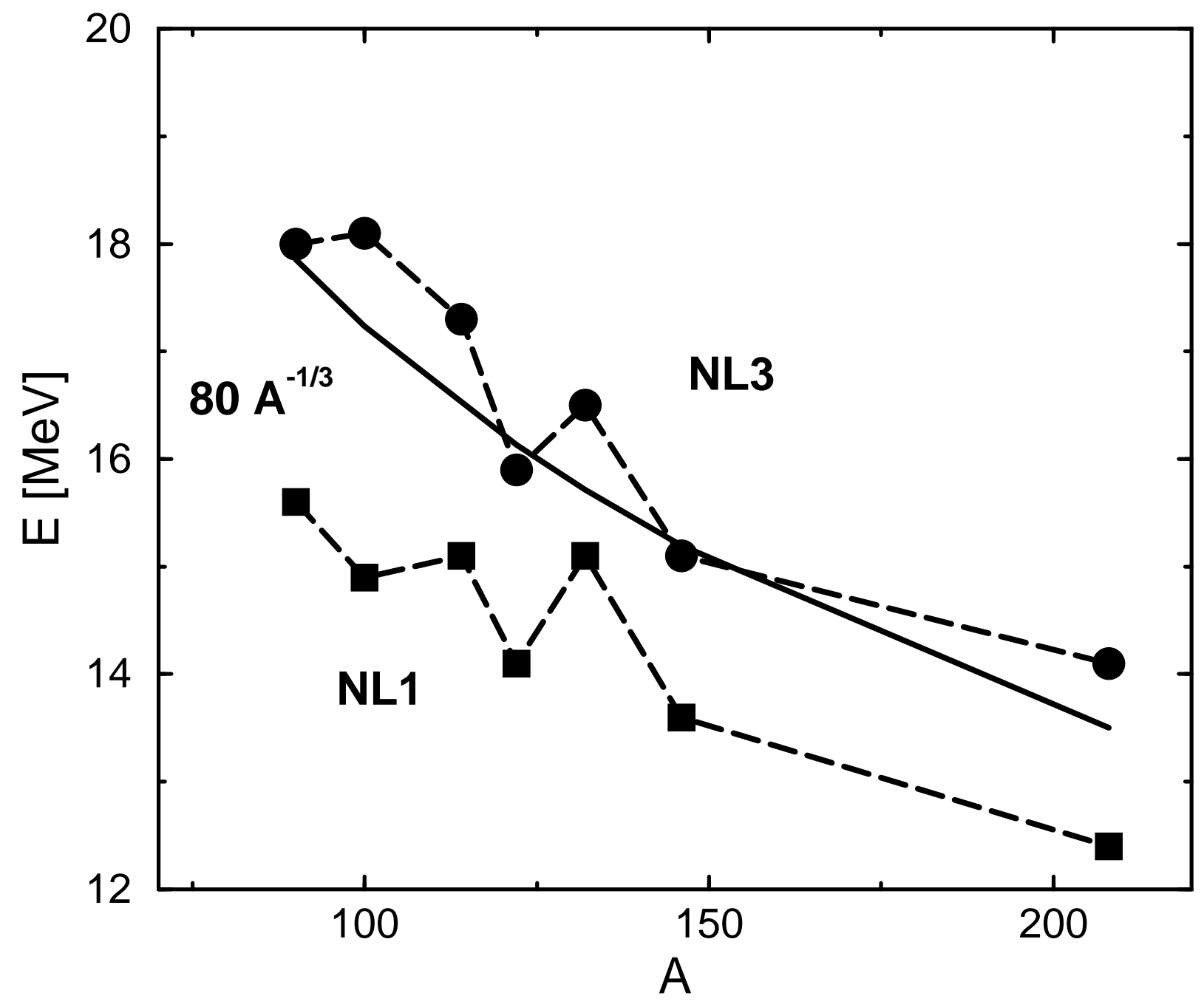



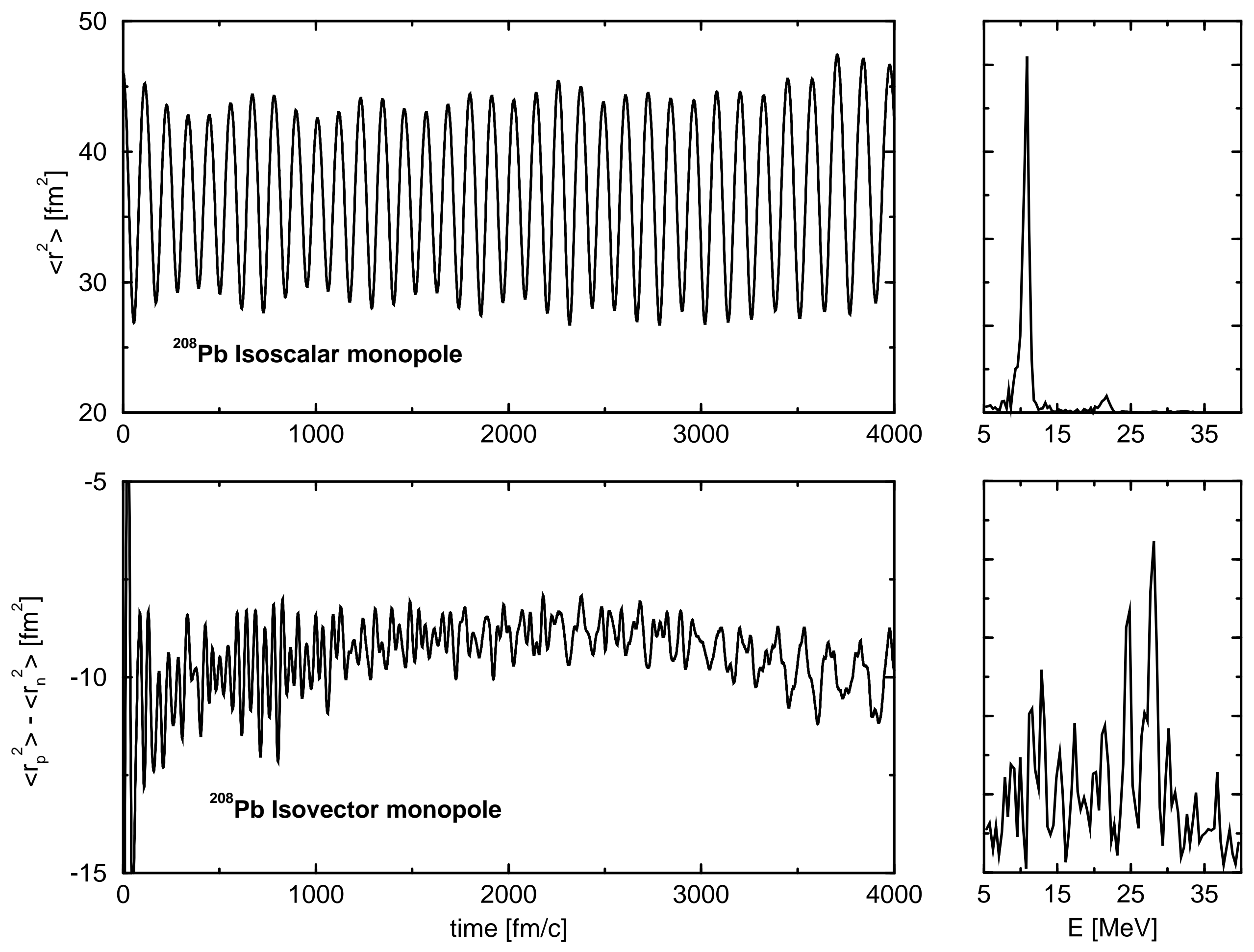


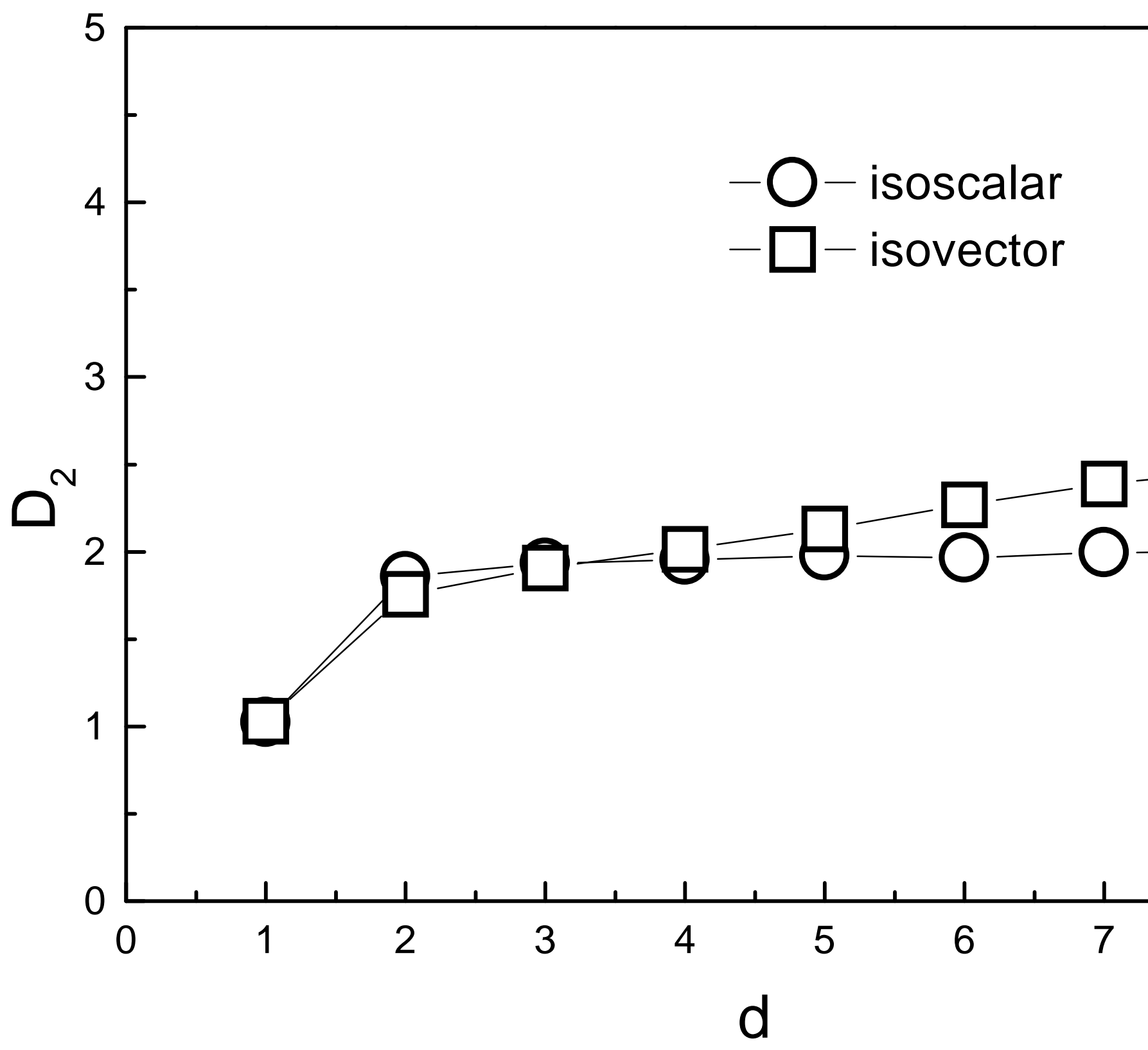




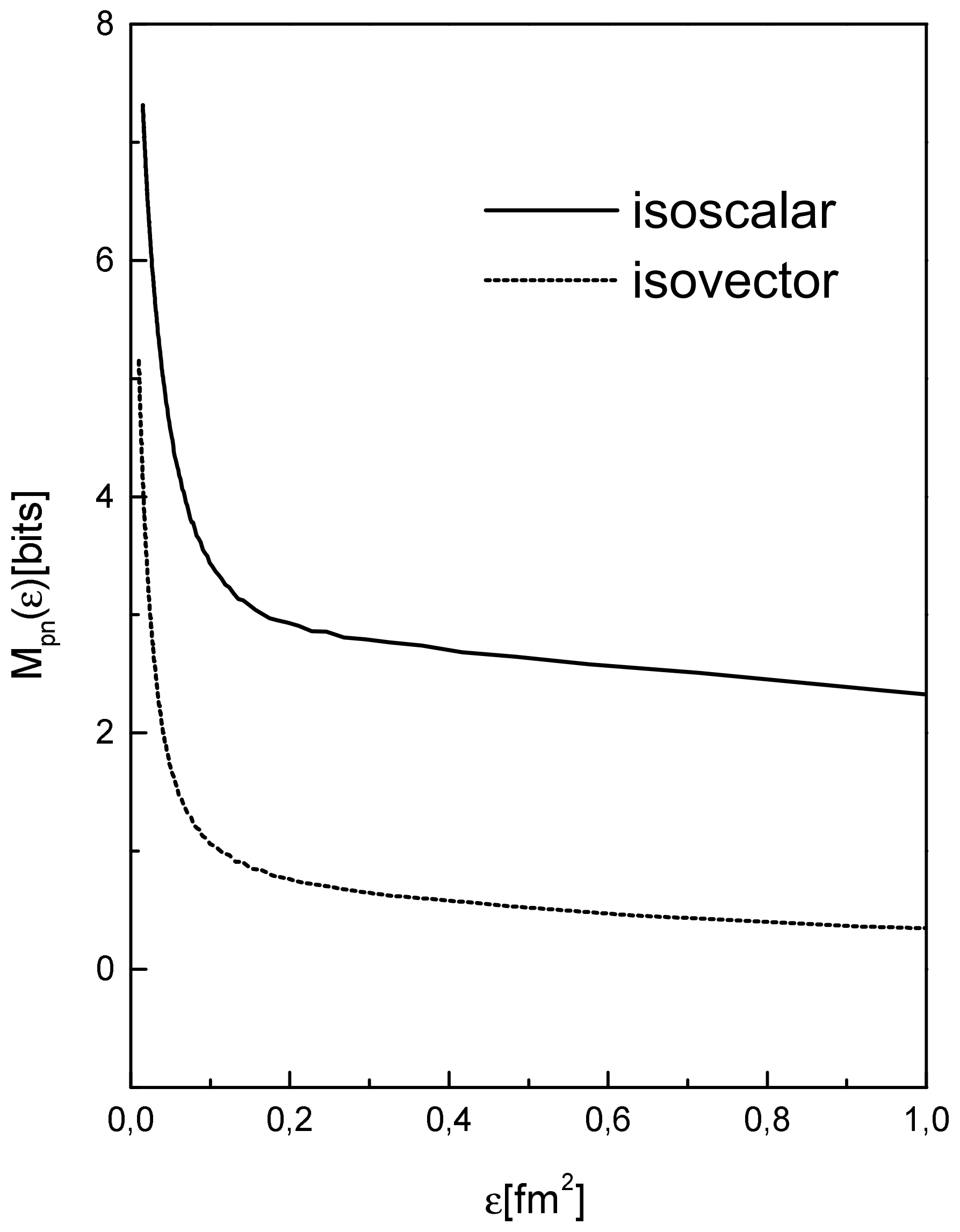

